\title{
Isospectral strings
}

H P W Gottlieb

School of Science, Griffith University, Nathan, Queensland 4111, Australia ${ }^{1}$

and

Department of Mathematics, University of Queensland, St. Lucia, Queensland 4072, Australia

${ }^{1}$ Permanent address, and address for correspondence

E-mail: H.Gottlieb@sct.gu.edu.au

April 2002 


\begin{abstract}
Transformations are found which give new density functions for strings isospectral to a given string, for the Dirichlet case. The formalism is also cast in a group-theoretic framework which unifies the procedure and enables invariant functions to be found. Several interesting examples of density functions for isospectral strings, including general power law forms, are given.
\end{abstract}




\section{Introduction}

Amongst vibrating systems, isospectral systems are those sharing the same set of eigenfrequencies. In an earlier paper [1] the author was primarily concerned with nonhomogeneous systems isospectral to homogeneous systems, e.g. nonuniform strings with harmonic frequency spectra as for the constant density case. In this paper, transformations yielding new density functions for strings isospectral to strings with any given density function are found, for the case of Dirichlet end conditions.

Isospectral systems are important in the study of inverse problems, since they are concrete realizations of the fact that the system is not uniquely determined by its eigenvalues: for strings, another set of values is required for unique reconstruction of the density. This may be another spectrum with different boundary conditions [2], or a set of nodal positions [3], [4], or eigenfunction norming constants. These matters have been reviewed by McLaughlin [5] and Gladwell [6, chapter 9], [7].

The present paper makes a simultaneous change of independent and dependent variables which leaves the governing differential equation unchanged in general form. Our work is quite different from the approach of Gladwell and Morassi [8], which is based upon the use of the Darboux Lemma after reduction of the governing equation to Sturm-Liouville form. They dealt mainly with longitudinal vibrations of rods, and only briefly, but with equal applicability, to strings. 


\section{Transformations}

The one-dimensional linearized wave equation for the transverse motion of a nonhomogeneous elastic string may be written in the form

$$
\frac{\partial^{2} v}{\partial \xi^{2}}=\phi(\xi) \frac{\partial^{2} v}{\partial t^{2}}
$$

where $v$ is the displacement, $t$ is the time, and $\phi(\xi)=(1 / \mathrm{T}) \rho(\xi)$, where $\rho(\xi)$ is the lineal density which may depend on the independent position variable $\xi$. Here, $\mathrm{T}$ is the tension; henceforth we shall absorb T into $\rho$ and simply refer to $\phi$ as the density.

We seek transformations to new coordinate $\mathrm{x}$ and new displacement $\mathrm{u}$ which preserve the structural form, but not the exact form, of the wave equation (2.1), and the Dirichlet boundary conditions of vanishing displacement at the ends. Let

$$
\mathrm{x}=\mathrm{x}(\xi) ; \quad v(\xi, \mathrm{t})=\gamma(\mathrm{x}) \mathrm{u}(\mathrm{x}, \mathrm{t})
$$

where $\gamma(x)$ is some positive nonsingular position-dependent but time-independent multiplicative function. Thus the Dirichlet (fixed end) boundary condition is preserved: $\mathrm{u}=0$ if and only if $\mathrm{v}=0$. We wish to find functions $\mathrm{x}(\xi)$ and $\gamma(\mathrm{x})$ such that the new displacement $u$ satisfies a nonhomogeneous wave equation

$$
\frac{\partial^{2} \mathrm{u}}{\partial \mathrm{x}^{2}}=\mathrm{f}(\mathrm{x}) \frac{\partial^{2} \mathrm{u}}{\partial \mathrm{t}^{2}}
$$


where $f(x)$ is some new density function, "dual" to the density function $\phi(\xi)$. Now $\partial^{2} v / \partial t^{2}=\gamma \partial^{2} u / \partial t^{2}$. If the time dependence in the displacement $v$ is assumed to be of the form $\exp (\mathrm{i} \omega \mathrm{t})$, where $\omega$ is the angular frequency of the transverse vibrations, then the new displacement $u$ will have the same time dependence with the same set of eigenfrequencies, and the two different strings with density functions $\phi$ and $f$ respectively will be isospectral for Dirichlet boundary conditions. Since the time variable is not transformed, the dependence of the displacement functions on t will henceforth be suppressed.

Denote differentiation with respect to $\mathrm{x}$ by a dash ' and differentiation with respect to $\xi$ by a grave $`$ Thus, for instance, $\partial v / \partial \xi \equiv v^{`} \equiv v^{\prime} x^{`}=x^{`}\left(\gamma u^{\prime}+\gamma^{\prime} u\right)$. Then

$$
\partial^{2} v / \partial \xi^{2}=\left(x^{\prime}\right)^{2} \gamma u^{\prime \prime}+\left[x^{\prime \prime} \gamma+2\left(x^{\prime}\right)^{2} \gamma^{\prime}\right] u^{\prime}+\left[x^{\prime \prime} \gamma^{\prime}+\left(x^{\prime}\right)^{2} \gamma^{\prime \prime}\right] u
$$

To obtain (2.3) from (2.4) and (2.1), first of all the coefficient of $u$ in (2.4) must vanish. This may be written as $0=\left(\gamma^{\prime} x^{\prime}\right)^{`} \equiv \gamma^{\prime}$. Thus the dependence of $\gamma$ on $\xi$ is immediately known as a linear form:

$$
\gamma(\mathrm{x}(\xi))=\text { constant } \xi \xi \text { constant } .
$$

(The constants are here written generically.)

Secondly, the coefficient of $u^{\prime}$ in (2.4) must vanish. This may be written as 0 $=(1 / \gamma)\left(\gamma^{2} x^{\prime}\right)^{`}$. Thus 


$$
\mathrm{dx} / \mathrm{d} \xi=\text { constant } / \gamma^{2}
$$

From (2.5), this gives (generically)

$$
\mathrm{x}(\xi)=(\text { constant } . \xi+\text { constant }) /(\text { constant } \xi+\text { constant })
$$

This is a (real) bilinear (Möbius) transformation. The inverse of such a coordinate transformation is again a bilinear transformation:

$$
\xi(\mathrm{x})=(\text { constant } . \mathrm{x}+\text { constant }) /(\text { constant } . \mathrm{x}+\text { constant })
$$

For convenience, the two strings will be taken as having equal length L:

$0 \leq \mathrm{x} \leq \mathrm{L}$. Furthermore, the end-point coordinates will be taken the same: when $\mathrm{x}=0$, $\xi=0$; when $\mathrm{x}=\mathrm{L}, \xi=\mathrm{L}$. There are then only two parameters left in the bilinear transformation relating $\mathrm{x}$ and $\xi$, but since the relation involves a quotient, there is then only one essential parameter. With a view towards group-theoretical considerations, the identity transformation $\xi=\mathrm{x}$ should correspond to the case when the parameter is zero. A suitable parametrization for (2.7b) (with $L \geq \xi \geq 0$ ) is

$$
\xi=(1+\mathrm{BL}) \frac{\mathrm{x}}{1+\mathrm{Bx}} \quad ; \quad \mathrm{B}>-\frac{1}{\mathrm{~L}}
$$

Note that the inverse of the coordinate transformation (2.8), namely 


$$
\mathrm{x}=\frac{\xi}{1+\mathrm{B}(\mathrm{L}-\xi)}
$$

may be written as

$$
\mathrm{x}=(1+\overline{\mathrm{B}} \mathrm{L}) \frac{\xi}{1+\overline{\mathrm{B}} \xi}
$$

which is of the same form as (2.8), where

$$
\overline{\mathrm{B}}=\frac{-\mathrm{B}}{1+\mathrm{BL}} .
$$

Whilst $\overline{\mathrm{B}}<0$ if $\mathrm{B}>0$, equation (2.10) preserves $\overline{\mathrm{B}}>-1 / \mathrm{L}$ if $\mathrm{B}>-1 / \mathrm{L}$. (These forms suggests an underlying group structure, as indeed there must be by consideration of the set of transformations which leave the structural form of the wave equation unchanged. This will be pursued in the next section.)

From (2.6), $\mathrm{d} \xi / \mathrm{dx}=$ constant. $\gamma^{2}$, so from (2.8) one arrives at $\gamma=$ constant/(1+Bx). Since a multiplicative constant in $\gamma$ does not affect the linear wave equation, there is no loss of generality in taking this last constant as unity. Thus

$$
\gamma(\mathrm{x})=\frac{1}{1+\mathrm{Bx}} .
$$

Notice that neither $\xi(\mathrm{x})$ nor $\gamma(\mathrm{x})$ depends on the density function. 
Finally, from the first term on the right side of (2.4) and using the wave equation (2.1), and noting (2.2b) and the fact that $x^{`} \equiv 1 / \xi^{\prime}$, comparison with the new wave equation (2.3) yields

$$
f(x)=\left(\frac{d \xi}{d x}\right)^{2} \phi(\xi(x))
$$

The above coordinate transformation result (2.8) now gives the sought-after transformation between density functions: the dual to $\phi$ is given by

$$
f(x)=\frac{(1+B L)^{2}}{(1+B x)^{4}} \phi\left(\frac{(1+B L) x}{1+B x}\right) .
$$

The transformation between the displacement functions is, from equations (2.2), (2.8) and (2.11),

$$
u(x)=(1+B x) v\left(\frac{(1+B L) x}{1+B x}\right)
$$

As mentioned above, the two string systems are isospectral, and this is independent of the value of B. Thus equations (2.13), (2.14), with (2.8b), represent a whole family of strings isospectral to (2.1). 


\section{Group-theoretical aspects}

The Lie group structure of the whole formalism may be conveniently studied by making a change of notation, first of all for $\mathrm{i}=1,2$, corresponding to Section 2 above, and then extending this to $i=3$ :

$$
\begin{aligned}
\frac{\partial^{2} u_{i}}{\partial x_{i}^{2}} & =f_{i}\left(x_{i}\right) \frac{\partial^{2} u_{i}}{\partial t^{2}} ; \\
x_{i}\left(x_{i+1}\right) & =\left(1+B_{i} L\right) \frac{x_{i+1}}{1+B_{i} x_{i+1}} ; B_{i}>-\frac{1}{L} ; \\
f_{i+1}\left(x_{i+1}\right) & =\frac{\left(1+B_{i} L\right)^{2}}{\left(1+B_{i} x_{i+1}\right)^{4}} f_{i}\left(\frac{\left(1+B_{i} L\right) x_{i+1}}{1+B_{i} x_{i+1}}\right) ; \\
u_{i+1}\left(x_{i+1}, t\right) & =\left(1+B_{i} x_{i+1}\right) u_{i}\left(\frac{\left(1+B_{i} L\right) x_{i+1}}{1+B_{i} x_{i+1}}, t\right)
\end{aligned}
$$

It may be noted that all of these transformations only involve substitutions: no integrals are involved here.

Consider first the coordinates themselves. From (3.2), the transformation $1 \rightarrow$

$2 \rightarrow 3$ gives overall

$$
x_{1}=\frac{\left(1+B_{1,2} L\right) x_{3}}{1+B_{1,2} x_{3}}
$$


where

$$
\mathrm{B}_{1,2}=\mathrm{B}_{1}+\mathrm{B}_{2}+\mathrm{B}_{1} \mathrm{~B}_{2} \mathrm{~L} .
$$

Regarding the B as the Lie group parameter, equation (3.5) confirms closure, since it is of the same form as (3.2); it can be confirmed that $\mathrm{B}_{1,2}>-1 / \mathrm{L}$. Equation (3.6) is the group parameter combination law. The identity transformation has $\mathrm{B}_{1}=0$. The inverse transformation parameter, for given $\mathrm{B}_{1}$, is just given by $\overline{\mathrm{B}}_{1}$ (c.f. (2.10)), when $\mathrm{B}_{1,2}=0$. Associativity can also be confirmed, using (3.6).

The group of transformations specified by the parameter B, being a onedimensional Lie group, is of course Abelian, as confirmed by (3.6): $B_{1,2}=B_{2,1}$. Any one-parameter group is equivalent to an additive group by suitable choice of parameter [9, p.294]. Let $\Psi\left(\mathrm{B}_{1}\right)=\left(\left.\left[\partial \mathrm{B}_{1,2} / \partial \mathrm{B}_{2}\right]\right|_{\mathrm{B}_{2}=0}\right)^{-1}$. Then the additive parameter is given by $\beta=\int_{0}^{B} \Psi\left(B_{1}\right) d B_{1}$. Here, $\Psi\left(B_{1}\right)=1 /\left(1+L_{1}\right)$, so

$$
\beta=(1 / \mathrm{L}) \log _{\mathrm{e}}(1+\mathrm{BL})
$$

with $\beta_{1,2}=\beta_{1}+\beta_{2}$. (Because of (2.8b), $\beta$ is real.) The correctness of this is readily confirmed by noting that the composition law (3.6) may be written as $\left(1+\mathrm{B}_{1,2} \mathrm{~L}\right)=$ $\left(1+B_{1} L\right)\left(1+B_{2} L\right)$, and taking logarithms. Thus $B=0$ iff $\beta=0$, and $B=[\exp (\beta L)-1] / L$. Because this exponential form is rather more complicated, the parametrization using $\mathrm{B}$ itself is actually more convenient for writing down transformations, and will continue to be used for the function transformations. 
From the relations (3.3), "closure" for the density functions can be confirmed.

$$
f_{3}\left(x_{3}\right)=\frac{\left(1+B_{1,2} L\right)^{2}}{\left(1+B_{1,2} x_{3}\right)^{4}} f_{1}\left(\frac{\left(1+B_{1,2} L\right) x_{3}}{1+B_{1,2} x_{3}}\right)
$$

where $\mathrm{B}_{1,2}$ is again given by equation (3.6). The result of two successive transformations is thus just another overall transformation. This has the important consequence that the dual of the dual of a density does not give another new family of isospectral densities, but is just another dual of the original system with appropriate parameter. It may be the same as the original system, but only if $B_{1,2}=0$, i.e. $B_{2}=\bar{B}_{1}$ (c.f. (2.10)). Thus if $f_{j}$ is dual to $f_{i}$, then $f_{i}$ is dual to $f_{j}$, so one may simply talk of dual densities. Similar statements concerning closure may be made for dual eigenfunctions, using the relations (3.4).

The transformed function on the right hand side of equation (3.3) has two components, a transformation of independent coordinate and a multiplying function. For infinitesimal transformations, the corresponding Lie group generator will therefore consist of two parts. There is a differential operator part, as is customary for purely coordinate transformations, but there is also a non-differential part containing only the coordinate itself, due to the multiplying function.

For a transformation with infinitesimal parameter $\delta \mathrm{B}$, the generator for transformations of functions given by (3.3), defined by $\mathrm{f}_{2}(\mathrm{x})=\left[1+\mathrm{i} \delta \mathrm{B} Q_{f}\right] \mathrm{f}_{1}(\mathrm{x})+$ $\mathrm{O}(\delta \mathrm{B})^{2}$, is found to be

$$
Q_{f}=-\mathrm{i}\left[2(\mathrm{~L}-2 \mathrm{x})+(\mathrm{L}-\mathrm{x}) \mathrm{x} \frac{\mathrm{d}}{\mathrm{dx}}\right] .
$$


A useful application of this Lie group formalism is to find invariants using the group generator (3.9). This enables one to find self-dual systems, i.e. systems which, under the transformations considered in this paper, remain exactly the same. Since the function remains unchanged, the condition in terms of the generator for a strictly selfdual function $\mathrm{g}(\mathrm{x})$ is

$$
Q_{f} \mathrm{~g}=0
$$

The resulting first-order linear differential equation is easily solved to give

$$
g(x)=\frac{\text { constant }}{[x(L-x)]^{2}}
$$

This might not have been so easily obtainable by inspection from (3.3). (It can be checked that $g_{2}(x)=g_{1}(x)$.) Regarded as a density function for $0 \leq x \leq L$, this function is obviously unphysical, being infinite at the end-points. There are therefore no physical strictly self-dual systems. Each physical density has dual densities different from itself, and the corresponding systems are distinct but isospectral. 


\section{Examples}

(i) The first and familiar case is that of a constant density k:

$$
\mathrm{f}_{1}\left(\mathrm{x}_{1}\right)=\mathrm{k},
$$

with harmonic eigenfrequencies

$$
\omega_{\mathrm{n}}=\mathrm{n} \pi /[\mathrm{L} \sqrt{\mathrm{k}}] ; \mathrm{n}=1,2,3, \ldots,
$$

and spatial eigenfunctions

$$
\mathrm{u}_{1}\left(\mathrm{x}_{1}\right)=\sin \left(\mathrm{n} \pi \mathrm{x}_{1} / \mathrm{L}\right) .
$$

The dual densities are given from (3.3) by

$$
f_{2}\left(x_{2}\right)=\frac{\left(1+B_{1} L\right)^{2} k}{\left(1+B_{1} x_{2}\right)^{4}}
$$

and the corresponding eigenfunctions from (3.4) are

$$
\mathrm{u}_{2}\left(\mathrm{x}_{2}\right)=\left(1+\mathrm{B}_{1} \mathrm{x}_{2}\right) \sin \left[\frac{\mathrm{n} \pi}{\mathrm{L}} \frac{\left(1+\mathrm{B}_{1} \mathrm{~L}\right) \mathrm{x}_{2}}{\left(1+\mathrm{B}_{1} \mathrm{x}_{2}\right)}\right] .
$$


The strings with these inverse-fourth-power type densities (4.4) are isospectral to the constant density case for all values of the parameter $\mathrm{B}_{1}>-1 / \mathrm{L}$, and their frequency spectrum is given by (4.2) (which is independent of $\mathrm{B}_{1}$ ). We shall call this the Borg string, since it was discussed by Borg [10, Section 20], who made a transformation to the normal Liouville form of second-order d.e. : the string isospectrality there only works for $\mathrm{f}_{1}=$ constant. It may be observed, however, that the functional forms of the multiplying function $\gamma(\mathrm{x})$ and the coordinate transformation $\xi(\mathrm{x})$ as appearing in this known example (4.5) are actually the same for the general formalism of this paper, and independent of the density, as noted after (2.11).

The $n-1$ internal nodes of the nth eigenfunction (4.5) corresponding to the dual densities (4.4) are given by

$$
\frac{\mathrm{x}_{2}{ }^{(\mathrm{N})}}{\mathrm{L}}=\frac{(\mathrm{N} / \mathrm{n})}{1+[1-(\mathrm{N} / \mathrm{n})] \mathrm{B}_{1} \mathrm{~L}} \quad, \quad \mathrm{~N}=1,2, \ldots, \mathrm{n}-1
$$

Unlike the uniform case of (4.3), the nodes here are not uniformly spaced, clearly distinguishing the eigenfunctions from those of the uniform case (cf. the discussion of uniqueness in the Introduction).

(ii) A less familiar but still explicitly solvable example is the case of inverse-square type density. This density will be called the Rayleigh string example, since it (but not its isospectral duals) was solved by Rayleigh [11, Section 142] (using different endpoint coordinates). Let

$$
f_{1}\left(x_{1}\right)=\frac{K_{2}}{\left(1+b x_{1}\right)^{2}}
$$

The eigenfunctions are 


$$
\mathrm{u}_{1}\left(\mathrm{x}_{1}\right)=\sqrt{1+\mathrm{bx}_{1}} \sin \left[\mathrm{N} \pi \frac{\log _{\mathrm{e}}(1+\mathrm{bx})}{\log _{\mathrm{e}}(1+\mathrm{bL})}\right],
$$

and the eigenfrequencies are

$$
\omega_{\mathrm{N}}=\frac{\mathrm{b}}{\sqrt{\mathrm{K}_{2}}} \sqrt{\frac{1}{4}+\frac{\mathrm{N}^{2} \pi^{2}}{\log _{\mathrm{e}}^{2}(1+\mathrm{bL})}} ; \quad \mathrm{N}=1,2,3, \ldots
$$

The dual density is given by

$$
f_{2}\left(x_{2}\right)=\frac{\left(1+B_{1} L\right)^{2} K_{2}}{\left(1+B_{1} x_{2}\right)^{2}\left(1+B_{C} x_{2}\right)^{2}}
$$

where

$$
\mathrm{B}_{\mathrm{C}}=\mathrm{b}+\mathrm{B}_{1}+\mathrm{bB}_{1} \mathrm{~L} .
$$

This looks like a composition (c.f. (3.6)) of two transformations with parameters b and $\mathrm{B}_{1}$, but here $\mathrm{b}$ is just a constant in (4.7), not a transformation parameter. (Note that in the special case with $\mathrm{b}=0$, i.e. $\mathrm{B}_{\mathrm{C}}=\mathrm{B}_{1}$, one is transforming a constant density case to the inverse fourth power density $\sim 1 /\left(1+\mathrm{B}_{1} \mathrm{x}_{2}\right)^{4}$, as for the density of the Borg string above; the eigenfrequencies (4.9) tend to the values like (4.2) as $b \rightarrow 0$.)

For general $b$ and $\mathrm{B}_{1}$, the eigenfunctions of the isospectral strings with dual densities (4.10) are 


$$
u_{2}\left(x_{2}\right)=\sqrt{\left(1+B_{1} x_{2}\right)\left(1+B_{C} x_{2}\right)} \sin \left[N \pi \frac{\log _{e}\left(\frac{1+B_{C} x_{2}}{1+B_{1} x_{2}}\right)}{\log _{e}(1+b L)}\right]
$$

with $\mathrm{B}_{\mathrm{C}}$ given by (4.11), and the eigenfrequencies are given by (4.9), independent of $\mathrm{B}_{1}$.

In the special case that $\mathrm{B}_{C}=0$ in (4.11), i.e. $\mathrm{B}_{1}=\overline{\mathrm{b}} \equiv-\mathrm{b} /(1+\mathrm{bL})(\mathrm{cf} .(2.10))$, then

$$
f_{2}\left(x_{2}\right)=K_{2} \frac{(1+\bar{b} L)^{2}}{\left(1+\bar{b} x_{2}\right)^{2}}=\frac{K_{2}}{\left[1+b\left(L-x_{2}\right)\right]^{2}}
$$

Such a density obviously has the same spectrum as (4.7) since it merely corresponds to reversing the spatial coordinate, i.e. "turning the string around" (c.f. [8] for the case of longitudinal vibrations of a rod). This is therefore a physically self-dual but not strictly self-dual (cf. section 3) string.

(iii) The case of a string with linear variation in density

$$
\mathrm{f}_{1}\left(\mathrm{x}_{1}\right)=\mathrm{K}_{1}\left(1+\mathrm{bx}_{1}\right)
$$

was studied for example by Fulcher [12]. The eigenfunctions involve Airy functions, and the frequencies are given by the roots of a rather complicated transcendental equations involving these functions. The dual densities are given by 


$$
f_{2}\left(x_{2}\right)=K_{1}\left(1+B_{1} L\right)^{2} \frac{\left(1+B_{C} x_{2}\right)}{\left(1+B_{1} x_{2}\right)^{5}},
$$

where $\mathrm{B}_{\mathrm{C}}$ is again given as in (4.11). These densities therefore in general behave like a linear form divided by a fifth-power form.

(iv) It is evident from the above examples that there is a general form for power law dependences. For density

$$
\left.\mathrm{f}_{1}\left(\mathrm{x}_{1}\right)=\mathrm{K}(1+\mathrm{bx})_{1}\right)^{\mathrm{n}}
$$

the eigenfrequencies depend on $\mathrm{K}$ and $\mathrm{b}$ and $\mathrm{n}$ (and $\mathrm{L}$ ). The dual densities are

$$
f_{2}\left(x_{2}\right)=K\left(1+B_{1} L\right)^{2} \frac{\left(1+B_{C} x_{2}\right)^{n}}{\left(1+B_{1} x_{2}\right)^{4+n}}
$$

where $B_{C}$ is given by equation (4.11), and the eigenfrequencies of the family of isospectral strings are the same as for (4.16), independent of $B_{1}$.

In the special case that $\mathrm{B}_{\mathrm{C}}=0$, i.e.

$$
\mathrm{B}_{1}=\overline{\mathrm{b}} \equiv-\mathrm{b} /(1+\mathrm{bL})
$$

then 


$$
f_{2}\left(x_{2}\right)=\frac{K(1+\bar{b} L)^{2}}{\left(1+\bar{b} x_{2}\right)^{4+n}}
$$

Here the constant $\bar{b}$, equation (4.18), is fixed by $b$ in (4.16): this is not a family. This means that for a string with density of linear form raised to some power $n$, there is (in addition to the general family (4.17)), one isospectral string with dual density just involving power -4-n.

(v) Other cases of known solutions of nonhomogeneous strings with spatially varying density may be invoked to yield new dual densities constructed as in Sections 1 or 2 above. For instance, Horgan and Chan [13], as well as mentioning densities corresponding to the "Borg" and "Rayleigh" strings described above (inverse fourth power and inverse square), give solutions and spectra for inverse linear dependence and for exponential dependence. (Their results appear to apply to the fundamental frequency only, but may evidently be extended to all frequencies by considering all the positive roots of the transcendental equations involving Bessel functions in [13].) These examples lead by the above procedures to new isospectral strings. 


\section{Discussion}

An explicit prescription for constructing densities dual to a given density has been given, allowing one to generate isospectral strings in the Dirichlet configuration. The dual densities, not distinguishable by the Dirichlet spectrum, could be distinguished by consideration of some other property, as outlined in the Introduction. For instance, the set of nodal points of $\mathrm{u}(\mathrm{x})$ will clearly not be the same as those of $v(\xi)$, according to equation (2.14). (See (4.6), for example.) Again, the case of a free end condition (Neumann boundary condition: the derivative of the function vanishes at the endpoint) is evidently not preserved by transformations of the type (2.2b), so eigenfunctions and eigenvalues for a free end will not be the same for densities which are dual with respect to fixed ends.

This raises the interesting question of finding some other transformation or approach which will produce densities dual to a given density when a free boundary condition is involved. For instance, is there a nonuniform string which is isospectral to a uniform string in the fixed-free configuration (Dirichlet boundary condition at one end and Neumann condition at the other), with spectrum proportional to the odd integers? 


\section{Acknowledgments}

The author thanks Professor Tony Bracken for several interesting and useful conversations. The hospitality of the Department of Mathematics, University of Queensland, St. Lucia, during an Academic Studies Program visit is gratefully acknowledged. 


\section{References}

[1] Gottlieb H P W 1988 Iso-spectral operators: Some model examples with discontinuous coefficients J. Math. Anal. Appl. 132 123-137

[2] Barcilon V 1983 Explicit solution of the inverse problem for a vibrating string $J$. Math. Anal. Appl. 93 222-234

[3] Shen C-L 1988 On the nodal sets of the eigenfunctions of the string equation SIAM J. Math. Anal. 19 1419-1424

[4] Shen C-L and Tsai T-M 1995 On a uniform approximation of the density function of a string equation using eigenvalues and nodal points and some related inverse nodal problems Inverse Problems 11 1113-1123

[5] McLaughlin J R 1986 Analytical methods for recovering coefficients in differential equations from spectral data SIAM Rev. 28 53-72

[6] Gladwell G M L 1986 Inverse Problems in Vibration (Dordrecht: Martinus Nijhoff)

[7] Gladwell G M L 1996 Inverse problems in vibration II Appl. Mech. Rev. 49 S25S34

[8] Gladwell G M L and Morassi A 1995 On isospectral rods, horns and strings Inverse Problems 11 533-554

[9] Hamermesh M 1989 Group Theory and its Application to Physical Problems (New York, Dover)

[10] Borg G 1946 Eine Umkehrung der Sturm-Liouvilleschen Eigenwertaufgabe Acta Math 78 1-96

[11] Rayleigh J W S 1945 The Theory of Sound, Vol. I (New York: Dover) 
[12] Fulcher L P 1985 Study of the eigenvalues of a nonuniform string Am. J. Phys. $53730-735$

[13] Horgan C O and Chan A M 1999 Vibration of inhomogeneous strings, rods and membranes J Sound Vib. 225 503-513 